\title{
La satisfacción con el tratamiento y la calidad de vida en pacientes con psoriasis pueden no estar directamente asociados. Estudio preliminar
}

\author{
Treatment satisfaction and quality of life in patients with psoriasis may not be directly \\ associated. Preliminary study
}

\begin{abstract}
Fernando Delgado-Borja1, Alain R. Rodríguez-Orozco*, Martha E. García-Pérez ${ }^{3}$, Jorge L. Pérez-Molina ${ }^{1}$, Christian Cortés-Rojo ${ }^{3}$ y Carlos Gómez-Alonso ${ }^{4}$

${ }^{1}$ Unidad de Medicina Familiar 80, Instituto Mexicano del Seguro Social; ${ }^{2}$ Facultad de Ciencias Médicas y Biológicas Dr Ignacio Chávez, Universidad Michoacana, San Nicolás de Hidalgo; ${ }^{3}$ Instituto de Investigaciones Quimicobiológicas, Universidad Michoacana, San Nicolás de Hidalgo; ${ }^{4}$ Centro de Investigaciones Biomédicas de Michoacán, Instituto Mexicano del Seguro Social. Morelia, Michoacán, México
\end{abstract}

En México se ha estimado recientemente una prevalencia de psoriasis del $2.9 \%{ }^{1}$. Esta es una enfermedad crónica compleja por la riqueza en su presentación clínica, por la variedad de opciones terapéuticas y también por el alto grado de insatisfacción de los pacientes con el tratamiento ${ }^{2}$. El objetivo de este trabajo preliminar fue evaluar la relación entre la calidad de vida y la satisfacción con el tratamiento en pacientes con psoriasis en una unidad de atención primaria. Fue un estudio transversal en el que participaron 41 pacientes. Se eligieron todos los pacientes que reunieran los siguientes criterios: mayores de 18 años de ambos sexos con diagnóstico confirmado de psoriasis en placa por un dermatólogo hasta el 1 de septiembre del año 2017 en una Unidad de Medicina Familiar del Instituto Mexicano del Seguro Social, sin comorbilidad, excepto diabetes mellitus controlada e hipertensión controlada en los últimos 6 meses según las guías de práctica clínica de estas enfermedades emitidas por la Secretaría de Salud de México. Todos los pacientes que aceptaron participar en el estudio firmaron el consentimiento informado. Se determinó la gravedad de la psoriasis con el Psoriasis Area and Severity Index (PASI). Para la evaluación de la calidad de vida relacionada con la enfermedad se utilizó el Psoriasis Disability Index (PDI) y para estimar la satisfacción de los pacientes con el tratamiento se utilizó el Cuestionario Español de Satisfacción de Tratamiento en Enfermos con Psoriasis (CESTEP) ${ }^{4}$.

El $43.9 \%$ de los pacientes del estudio fueron mujeres y el $56.1 \%$ fueron hombres, con una edad promedio de $48.49 \pm 13.92$ años. El índice de masa corporal fue de $28.32 \pm 4.28$. El tiempo de evolución de la enfermedad fue de $11.07 \pm 7.83$ años. De acuerdo con el PASI, presentaban psoriasis leve el $65.4 \%$ de los pacientes y moderada el $34.6 \%$. Entre ellos, el $14.6 \%$ padeció algún brote en el último mes.

Llama la atención que el $87.8 \%$ de los pacientes estuvieron satisfechos con su tratamiento y que el $80.5 \%$ refirieron tener buena calidad de vida, pero no se encontró asociación entre estas variables $\left(\chi^{2}=1.380 ; p=0.051\right)$ (Tabla 1). Tampoco se encontró relación entre la adherencia terapéutica y la satisfacción con el tratamiento $(p=0.061)$, pues algunos pacientes se mostraron satisfechos con el tratamiento a pesar de no mostrar adherencia total a este, y modificaron los tiempos e intervalos de aplicación de los medicamentos; es necesario estudiar las razones que motivaron estas conductas.

\section{Correspondencia:}

*Alain R. Rodríguez-Orozco

Rafael Carrillo esquina Salvador González Herrejón s/n

Bosque Cuauhtemoc, Col. Centro

Fecha de recepción: 05-06-2020

Fecha de aceptación: 15-07-2020

E-mail: alain.rodriguez@umich.mx

0009-7411/@ 2020 Academia Mexicana de Cirugía. Publicado por Permanyer. Este es un (http://creativecommons.org/licenses/by-nc-nd/4.0/).
Cir Cir. 2021;89(6):846-847

Contents available at PubMed www.cirugiaycirujanos.com artículo open access bajo la licencia CC BY-NC-ND 
Tabla 1. Calidad de vida y satisfacción con el tratamiento en pacientes con psoriasis

\begin{tabular}{lcc}
\hline Calidad de vida (PDI) & \multicolumn{2}{c}{ Grado de satisfacción (CESTEP) } \\
\cline { 2 - 3 } & Satisfecho (\%) & No satisfecho (\%) \\
\hline Buena calidad de vida & $28(68.30)$ & $5(12.2)$ \\
Regular calidad de vida & $6(14.6)$ & 0 \\
Mala calidad de vida & $2(4.9)$ & 0 \\
Total & $36(87.8)$ & $5(12.2)$ \\
\hline
\end{tabular}

CESTEP: Cuestionario Español de Satisfacción con el Tratamiento del Paciente con Psoriasis; PDI: Psoriasis disability index.

$\chi^{2}: 1.380 ;$ grado de libertad: $2 ; p=0.051$

A pesar de existir un vasto arsenal terapéutico para tratar a los pacientes con psoriasis, se ha establecido que más del $70 \%$ de ellos no están satisfechos con su tratamiento y que llega a ser alta la discordancia entre la opinión del médico y la del paciente respecto a la percepción de gravedad de la enfermedad ${ }^{2}$. De acuerdo con otros autores, con frecuencia no hay una asociación clara entre el impacto de la enfermedad, la percepción de bienestar y la gravedad de la psoriasis estimada con el PASI ${ }^{5}$.

\section{Financiamiento}

Los autores declaran haber recibido financiamiento por parte de la Coordinación de la Investigación Científica, Universidad Michoacana de San Nicolás de Hidalgo, 2019-2020.

\section{Conflicto de intereses}

Los autores declaran no tener ningún conflicto de intereses.

\section{Responsabilidades éticas}

Protección de personas y animales. Los autores declaran que para esta investigación no se han realizado experimentos en seres humanos ni en animales.

Confidencialidad de los datos. Los autores declaran que han seguido los protocolos de su centro de trabajo sobre la publicación de datos de pacientes.

Derecho a la privacidad y consentimiento informado. Los autores han obtenido el consentimiento informado de los pacientes y/o sujetos referidos en el artículo. Este documento obra en poder del autor de correspondencia.

\section{Bibliografía}

1. Esquivel-García R, Estévez-Delgado G, Rodríguez-Orozco AR, Ochoa-Zarzosa A, García-Pérez ME. La psoriasis: de la investigación básica y clínica al desarrollo de nuevos tratamientos. Gac Med Mex. 2018;154:502-8.

2. Ribera-Pibernat M, Caballé G, Fernández-Chico N, Fuente MJ, Ferrándiz C. Impacto de la psoriasis en la calidad de vida del paciente español. Piel. 2004;19:242-9

3. Finlay AY, Kelly SE. Psoriasis: an index of disability. Clin Exp Derm. 1987; 12:8-11.

4. Ribera M, Daudén E, Puig L, Briones VG, Herranz JM, Bordas X, et al.; Grupo de Estudio NEODERMA. [Design and validation of a questionnaire to measure treatment satisfaction in patients with moderate-to-severe psoriasis: the NEODERMA study]. Actas Dermosifiliogr. 2011;102:28-38.

5. Reimus JL, Vingerhoets AJ, Soons PH, Korstanje MJ. Suffering in psoriasis patients: its relation with illness severity and subjective well-being. Int J Dermatol. 2007;46:1042-5. 